\section{INTUSSUSCEPTION ENCEPHALOPATHY: A CLINICALLY DECEPTIVE PRESENTATION}

Maymunah Khries*, Paul Bellis, Yincent Tse, Leigh McDonald. Great North Children's Hospital

10.1136/archdischild-2021-europaediatrics.342

A 7-month-old presented with reduced responsiveness and non-bilious vomiting. On presentation, he was encephalopathic, apyrexial with normal vital signs. Pupils were intermittently miotic and initial abdominal examination was normal. Investigations including blood gas, biochemistry, inflammatory markers, metabolic and toxicology panels were normal. A CT brain scan showed no abnormality.

Abdominal examination 24 hours later elicited a possibility of tenderness.

Therefore we proceeded with abdominal ultrasound which revealed an evidence of ileocolic intussusception. Initial standard management of ileocolic intussecption was attempted by radiological pneumatic reduction (air enema) which was unsuccessful. Subsequent definitive surgical management achieved by laparotomy and manual reduction successfully released the obstruction. He recovered uneventfully and underwent usual post-surgical care.

Discussion This case illustrates a rare occasion of intussusception presenting as an acute encephalopathy in the absence of typical signs of bowel obstruction.

Although uncommon, the recognition of this possibility should be entertained, particularly in an unexplained encephalopathy.

A recent study showed about $4 \%$ of children diagnosed with intussusception had one or more neurological symptoms recorded at presentation. Lethargy was the most frequent, followed by hypotonia, generalised weakness, paroxysmal events, and fluctuating consciousness. One study reported a series of 13 cases of children whom impairment of the mental state preceded the appearance of common gastrointestinal symptoms. Another distinctive feature is the presence of miosis. The aetiology is unclear but there has been hypotheses that this could be caused by the production of endogenous opioid in response to stress and pain.

In conclusion, the incidence of children with bowel aetiology having an altered mental status as a primary presentation is rare. However, as prognosis of intussusception can be timedependent, early recognition of this possibility by clinicians is crucial in order to minimise serious morbidity and mortality risk.

\section{LUPA - A PILOT PROJECT DURING THE COVID-19 PANDEMIC}

João Sousa Marques*, Inês Silva Costa, Clara Gomes, Lígia M Ferreira, Sofia Reis, Cristina Baptista. Pediatrics Department, Centro Hospitalar Tondela-Viseu EPE, Portugal

10.1136/archdischild-2021-europaediatrics.343

The COVID-19 pandemic changed the way we look at healthcare management and had a great impact in the social dynamics related to disease awareness and search for acute care. During the initial lockdown, we implemented a pilot project in the emergency department (ER) that consisted on a telephonic line named Linha de Urgência Pediátrica de Apoio
(LUPA), run by interns, to who parents could call when their kids had acute disease.

The goal of this study is to identify the contribution of this project to avoid unnecessary admissions in the ER during this pandemic period and to assess the effectively needed admissions.

We overviewed, restrospectively, the records of all phone calls received between March and October 2020 and ran a frequency analysis of the results.

During the defined time period, we received 288 phone calls, equally distributed between Monday to Sunday and 9am to $9 \mathrm{pm}$. Mothers accounted for $90 \%$ of the callers.

From the total amount of patients, 54\% were females with a median age of 20 months and a median time of illness of 2 days. The most frequent reasons for calling LUPA were fever (35\%), cutaneous alterations (18\%) and cough (8\%). Fever was predominantly associated with diarrhea (18\%) but in $29 \%$ of cases was the only reported symptom. Active surveillance of warning signs at home was the most chosen conduct, in $71 \%$ of cases. Admission to the ER was advised in $26 \%$ of cases.

LUPA was a usefull resource during the COVID 19 pandemic and helped managing benign and self-limited conditions, which are frequent in pediatric acute care.

\section{PROGNOSIS AND RISK FACTORS OF PATIENTS WITH ACUTE KIDNEY INJURY IN THE PICU}

Ilayda Altun*, Fatih Aygün, Nur Canpolat, Alper Kaçar. Istanbul University-Cerrahpasa, Cerrahpasa School of Medicine, Department of Child Health and Diseases

10.1136/archdischild-2021-europaediatrics.344

Acute kidney injury is an independent risk factor for morbidity and mortality in critically ill children in the pediatric intensive care (PICU).

This study was conducted to assess the frequency of acute kidney injury(AKI) and risk factors associated with AKI in (PICU) patients.

This retrospective study was conducted in two PICUs serving children under the age of 18 . The data of all patients admitted to the PICU for various critical illnesses between January 2014 and June 2019 were extracted from electronic and written medical records and the patients with AKI on admission were included in this study. Demographic data and reason for hospitalization were recorded. The patients' sex, age, invasive or non-invasive mechanical ventilation requirement, duration of hospitalization in the PICU and mortality were recorded. In addition, vital signs, CBC parameters and biochemistry results, the paediatric risk of mortality

(PRISM) score were recorded on admission. The patients were compared according to whether they had AKI and mortality.

Between January 2014 and July 2019, a total of 1,107 children were admitted to the PICU. The most common primary diagnosis was sepsis. Of them, 243

(22.0\%) had AKI during PICU admission. Fifty-three patients with AKI (21.8\%) died. Of them, 21 patients died because of septic shock. Among the patients with AKI, survivors required fewer inotropic drug usage $(\mathrm{P}<0.001)$, CRRT $(\mathrm{P}=0.047)$ and mechanical ventilation $(\mathrm{P}<0.001)$ compared to the patients who died. According to logistic regression analysis, the ORs were as follows: 
6.027 (95\% CI, 3.996-9.090) for inotropic drug usage, 2.348 (95\% CI, 1.547-3.564) for RBC transfusion requirement, 1.655 (95\% CI, 1.162-2.356) for RDW $>15.1 \%$ and 2.950 (95\% CI, 1.933-4.503) for PLT < $150 \times 103 / \mathrm{uL}$.

The AKI is associated with prolonged hospitalization and increased mortality.among critically ill children. In patients with AKI, the need for mechanical ventilation, CRRT and inotropic drugs was statistically associated with mortality.

\section{SPICING UP THE EDMONTON ANTICOAGULATION PROTOCOL: INTERFERENCE OF DIETARY CURCUMIN ON COAGULATION STATUS DURING MECHANICAL CIRCULATORY SUPPORT WITH BERLIN HEART LVAD}

Vanja Zvonar*, S Dessardo, F Rubić, S Galić, M Cvitković, T Matić, H Matković. University Hospital Centre Zagreb

\subsection{6/archdischild-2021-europaediatrics.345}

In patients with implanted Berlin Heart EXCOR ${ }^{\circledR}$ Ventricular Assist Device, appropriate anticoagulation and antiaggregation within pre-defined values are essential for proper device function, and vital in order to avoid complications such as thromboembolic events and/or bleeding.

Important factors that contribute to achieve the desired coagulation control are patient's pharmacogenomics, liver status with nutritional status and interactions with other medications and supplements given to the patient during treatment.

Bleeding or clotting issues that could occur are addressed according to aetiology, site of the incident, laboratory and clinical parameters.

We describe a 3-year-old female patient with restrictive cardiomyopathy and acute heart failure subjected to implantation of Berlin Heart EXCOR ${ }^{\circledR}$ paediatric Left Ventricular Assist Device mechanical support as bridge therapy to cardiac transplantation. After device implantation, anticoagulation and antiaggregation were started and maintained according to the Edmonton Anticoagulation and Platelet Inhibition Protocol. Achievement of appropriate anticoagulation was compromised due to a non-disclosed addition of turmeric in her dietary regimen by parents. Curcumin, a polyphenol responsible for the yellow colour of turmeric, possesses anticoagulant properties, prolongs aPTT and PT significantly and inhibits thrombin and FXa activities. As a result, unexpected oscillations in the coagulation profile occurred, which represented a substantial management challenge.

The aim of this report is to analyse and discuss the factors that could have been contributed to the difficult control of anticoagulation in our patient, with emphasis on the potential danger of undetected compounds deriving from sub-optimal control of paediatric patients during parental presence in PICU.

\section{BIVALIRUDIN USE DURING CONTINUOUS RENAL REPLACEMENT THERAPY IN A 2.5-YEAR-OLD GIRL SUFFERING FROM CARDIOMYOPATHY AND RECEIVING VAD SUPPORT}

Hana Matković*, Jasna Slaviček, Filip Rubić, Sandro Dessardo, Miran Cvitković, Toni Matić, Dorotea Bartoniček, Dražen Belina, Ivanka Kos, Slobodan Galić. Department of Paediatrics, University Hospital Centre Zagreb; School of medicine, University of Zagreb
Ventricle assist devices (VAD) are often the only bridge to heart transplantation in children with deteriorating cardiomyopathies.

Berlin Heart EXCOR Pediatric is registered for children and can be used to support the function of right, left or both ventricles. Despite the use of the 'Edmonton protocol' to guide the anticoagulation management, thromboembolism and hemorrhage are common adverse effects. Moreover, prolonged intravenous heparin use is often complicated with heparin induced thrombocytopenia (HIT), which leads to a conclusion that new antithrombotic strategies are needed, such as use of direct inhibitor of thrombin, i.e. bivalirudin.

In our patient, a 2.5 -year-old girl with restrictive cardiomyopathy, heart decompensation occurred after a viral infection (with later confirmation of parvovirus B19 myocarditis). Mechanical circulatory support with VA ECMO was started, with an implantation of LVAD Berlin Heart 3 weeks later. She spent 125 days on VAD support waiting for a heart transplantation. The 'Edmonton protocol' was followed for anticoagulation and anti-aggregation therapy guidance (warfarin, acetylsalicylic acid and clopidogrel). She suffered multiple complications: bilateral pleural effusions, middle cerebral artery thrombosis, fibrin over the VAD cannula, intracranial hemorrhage and shunt meningitis. During an episode of septic shock, acute renal failure occurred, requiring continuous venovenous hemodiafiltration that has been continued for 21 days. Prolonged thrombocytopenia led to diagnosis of HIT, so heparin was replaced with bivalirudin (direct thrombin inhibitor) as anticoagulation therapy, in addition to acetylsalicylic acid and clopidogrel. Bivalirudin use is easily monitored by APTT and ACT and the dosage is modified according to it. Bivalirudin has been used for 16 days along with CVVHDF, without hemofilter occlusion or fibrin deposits on cannulas of $\mathrm{BH}$ Excor.

According to our experience and cases described in literature, bivalirudin has been used in children with VAD Berlin Heart EXCOR with positive outcomes. In addition, successful use of bivalirudin for anticoagulation during continuous renal replacement was associated with longer hemofilter survival time and less hemorrhagic and/or thrombotic events. On the other hand, there are some disadvantages such as lack of pediatric experience, no available antidote and high price. From all of the above, we may conclude that new experiences and investigations are needed and alternative anti-thrombotic drugs, such as bivalirudin, should be considered for patients with VAD and CVVHDF.

\section{HYPERFREQUENT USERS OF THE EMERGENCY DEPARTMENT DURING THE COVID-19 PANDEMIC - A SURVEY STUDY}

João Sousa Marques*, Ana Gisela Oliveira, Inês Costa, Sofia Reis, Clara Gomes, Cristina Baptista. Centro Hospitalar Tondela Viseu, Pediatrics, Viseu, Portugal

\subsection{6/archdischild-2021-europaediatrics.347}

Hyperfrequent users (HU) of the pediatric emergency department (ED) is a group of patients that tendentially play an important stress factor in health services but there is no data available yet to the role of their behaviour during the COVID-19 pandemic - the purpose of our study.

A quota sampling out of 1816 HU from a Level II Hospital, defined by $\geq 10$ admissions in a single civil year, between 